\title{
Benefit from ifosfamide treatment in small-cell lung cancer: A meta-analysis
}

\author{
HUIZHEN YANG ${ }^{1}$, YUN MA $^{1}$, ZHIDA LIU $^{1}$, ZHENG WANG $^{1}$, BAOHUI HAN $^{2}$ and LIJUN MA ${ }^{1}$ \\ ${ }^{1}$ Department of Respiratory and Critical Care Medicine, People's Hospital of Zhengzhou University, \\ Zhengzhou, Henan 450003; ${ }^{2}$ Department of Pulmonary Medicine, Shanghai Chest Hospital, \\ Shanghai Jiaotong University, Shanghai 200030, P.R. China
}

Received September 16, 2014; Accepted November 5, 2014

DOI: $10.3892 / \mathrm{mco} .2014 .467$

\begin{abstract}
This study was conducted to compare the efficacy and safety of ifosfamide plus etoposide plus platinum (IEP) to that of etoposide plus platinum (EP) in patients with previously untreated small-cell lung cancer (SCLC). The Cochrane Library, Embase, MEDLINE and Chinese Biomedical Literature databases were searched to identify all randomized controlled trials comparing IEP to EP in patients with histologically proven SCLC. Two investigators independently assessed the quality of the relevant trials and extracted data. We analyzed the data using Review Manager software, version 4.2.8. A total of 4 trials with 447 previously untreated SCLC patients were included in this meta-analysis. The results of the meta-analysis indicated that there were no significant differences in the overall response [relative risk $(\mathrm{RR})=1.07,95 \%$ confidence interval $(\mathrm{CI})$ : 0.97-1.19], 1-year survival rate $(\mathrm{RR}=1.22,95 \% \mathrm{CI}$ : 0.96-1.55) and 2-year survival rate $(\mathrm{RR}=1.52,95 \% \mathrm{CI}: 0.75-3.07)$ between the IEP and $\mathrm{EP}$ regimens. However, there were significant differences between the IEP and EP regimens regarding the incidence of grade $3 / 4$ neutropenia $(\mathrm{RR}=1.52$, 95\% $\mathrm{CI}$ : $1.07-2.17)$ and grade $3 / 4$ vomiting ( $\mathrm{RR}=1.78$, 95\% CI: 1.02-3.11). In conclusion, our results suggested that IEP is not superior to EP regimen for the treatment of previously untreated SCLC, whereas the IEP regimen is associated with more severe hematological and gastrointestinal toxicities compared to EP. Therefore, the use of ifosfamide in multimodality treatment regimens requires further investigation.
\end{abstract}

Correspondence to: Professor Lijun Ma, Department of Respiratory and Critical Care Medicine, People's Hospital of Zhengzhou University, 7 Weiwu Road, Jinshui District, Zhengzhou, Henan 450003, P.R. China

E-mail: malijun0401@163.com

Key words: etoposide, platinum, ifosfamide, small-cell lung cancer

\section{Introduction}

Small-cell lung cancer (SCLC) accounts for $\sim 15 \%$ of all lung cancers (1) and is characterized by a rapid tumor growth rate and early dissemination to regional lymph nodes and to distant sites (2). The emergence of chemotherapy has significantly improved quality of life and survival in SCLC patients. However, long-term survival in SCLC patients has not been satisfactory (3-5). Etoposide plus platinum (EP) is currently the standard first-line treatment used in SCLC to obtain longer overall survival (OS) and progression-free survival, although numerous chemotherapeutic regimens comprising various drugs have been investigated and tested in clinical trials $(6,7)$.

Ifosfamide is considered to be effective for SCLC, even when administered alone (8). Previous studies demonstrated that regimens including ifosfamide may achieve comparable response rates, survival and safety with the standard chemotherapy regimens for SCLC (9-12). Loehrer et al (13) and Miyamoto et al (14) were the first to apply ifosfamide in combination with the EP regimen (IEP) for the treatment of SCLC. Particularly since the Hoosier Oncology Group initiated a pilot trial to evaluate the efficacy of IEP in extensive SCLC, reporting encouraging results in terms of complete response rate (CRR) and median survival time (15), several clinical trials were performed to further confirm the antitumor activity of ifosfamide in SCLC when used in combination with standard chemotherapeutic agents (16-20). Although discrepant conclusions have been reported by different trials, the IEP regimen is generally applied in SCLC patients according to the clinician's decision.

A meta-analysis reported that there was no strong clinical evidence indicating an advantage of other platinum-based regimens when compared to the EP regimen for patients with extensive SCLC requiring chemotherapy. Moreover, a clinical study comparing EP to the IEP regimen in SCLC was included in the meta-analysis, with the IEP group exhibiting no significant superiority to the EP group (6).

It has not been clearly determined whether the addition of ifosfamide to standard regimens is necessary. In order to evaluate the advantages and disadvantages of ifosfamide for SCLC, we systematically searched all available published randomized controlled trials (RCTs) comparing IEP and EP in SCLC. The aim of this meta-analysis was to synthesize all 
Table I. Characteristics of the 4 trials comparing ifosfamide + etoposide + platinum with etoposide + platinum in patients with previously untreated small-cell lung cancer.

\begin{tabular}{lccccccc}
\hline Author & $\begin{array}{c}\text { Sample } \\
\text { size }\end{array}$ & Randomization & $\begin{array}{c}\text { Allocation } \\
\text { concealment }\end{array}$ & Blinding & $\begin{array}{c}\text { Completeness } \\
\text { of follow-up }\end{array}$ & Quality & (Refs.) \\
\hline Miyamoto et al & 92 & Adequate & Unclear & Unclear & Adequate & C & $(14)$ \\
Loehrer et al & 171 & Adequate & Adequate & Unclear & Adequate & B & $(15)$ \\
Zhou et al & 64 & Adequate & Unclear & Unclear & Adequate & C & $(19)$ \\
Wu et al & 120 & Adequate & Unclear & Unclear & Adequate & C & $(20)$ \\
\hline
\end{tabular}

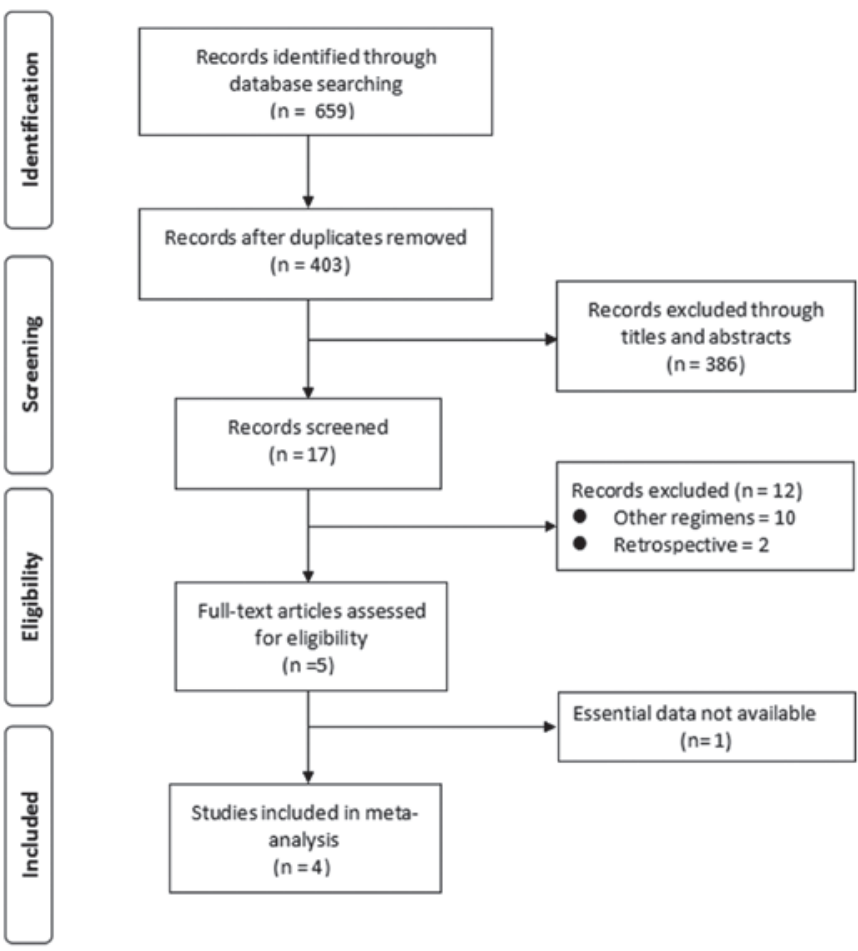

Figure 1. Flowchart of study selection process. The search identified trials comparing ifosfamide + etoposide + platinum to etoposide + platinum in the treatment of small-cell lung cancer.

evidence originating from direct comparisons and assess the efficacy and tolerability of the two regimens used as standard first-line treatment for previously untreated SCLC.

\section{Materials and methods}

Search strategy. The Cochrane Library, Embase, MEDLINE and Chinese Biomedical Literature databases were searched to identify all RCTs comparing IEP to the EP regimen in patients with histologically proven SCLC. The following keywords were used: 'small-cell lung cancer' or 'small-cell lung carcinoma', 'etoposide' and 'ifosfamide'. The search was limited to 'randomized controlled trials'. No limits based on language were imposed.

Selection of studies. Two investigators (H.Z.Y. and Y.M.) independently assessed the retrieved articles. Any disagreements were resolved by consensus. The studies included in the meta-analysis were required to meet all the following criteria: i) the IEP and EP regimens were compared in previously untreated SCLC patients; and ii) essential information for the meta-analysis was reported in the RCTs. The study selection process is summarized in Fig. 1.

Data extraction. The same investigators (H.Z.Y. and Y.M.) independently extracted relevant data on study characteristics and examination results by using a standardized form. To resolve any disagreements between the reviewers, a third reviewer (L.J.M.) assessed all discrepant items and the majority opinion was used for analysis.

The quality items assessed were randomization, allocation concealment, blinding (participants, investigators, outcome assessors and data analysis) and completeness of follow-up.

Outcome measures. The efficacy outcomes were CRR, objective response rate and survival rate. Adverse effects included grade $3 / 4$ hematological toxicity and grade $3 / 4$ gastrointestinal toxicity.

Statistical analysis. Review Manager software, version 4.2.8 (The Cochrane Collaboration) was used for statistical analyses. Relative risks (RRs) and their 95\% confidence intervals (CIs) were calculated using the Mantel-Haenszel method for dichotomous outcomes. We estimated heterogeneity between trials by using the Cochran's Q statistic test and the $\mathrm{I}^{2}$ metric. The fixed-effects model was generally used for the calculations, unless there was significant heterogeneity, in which case the random-effects statistical model was applied. To assess the sources of possible variation in the study results, we performed a subgroup analysis. Descriptive techniques were employed to assess adverse effects.

\section{Results}

Search results. Our search yielded 659 primary studies, of which 256 were duplicate trials, 10 trials referred to treatments other than IEP or EP and 2 retrospective trials were detected. Of the 5 trials considered eligible for assessment, 1 was excluded due to lack of essential data for the meta-analysis. Finally, 4 RCTs, totaling 447 patients, were included in this meta-analysis (Fig. 1). The quality and characteristics of the 4 included studies are summarized in Table I.

\section{Meta-analysis results}

Response. Response rate was reported in the 4 trials $(14,15,19,20)$ and the meta-analysis indicated that IEP was superior to the 


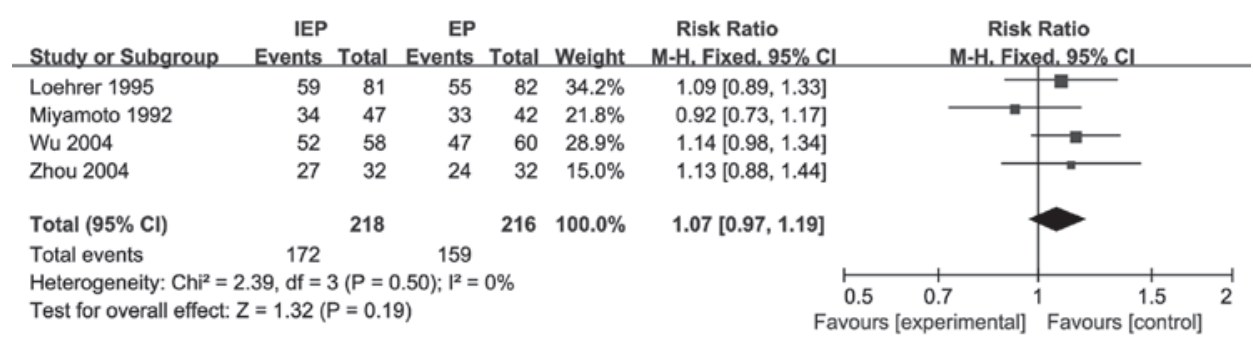

Figure 2. Meta-analysis of overall response of patients with small-cell lung cancer who were treated with ifosfamide + etoposide + platinum (IEP) vs. those treated with the etoposide + platinum (EP) regimen. $\mathrm{CI}$, confidence interval.

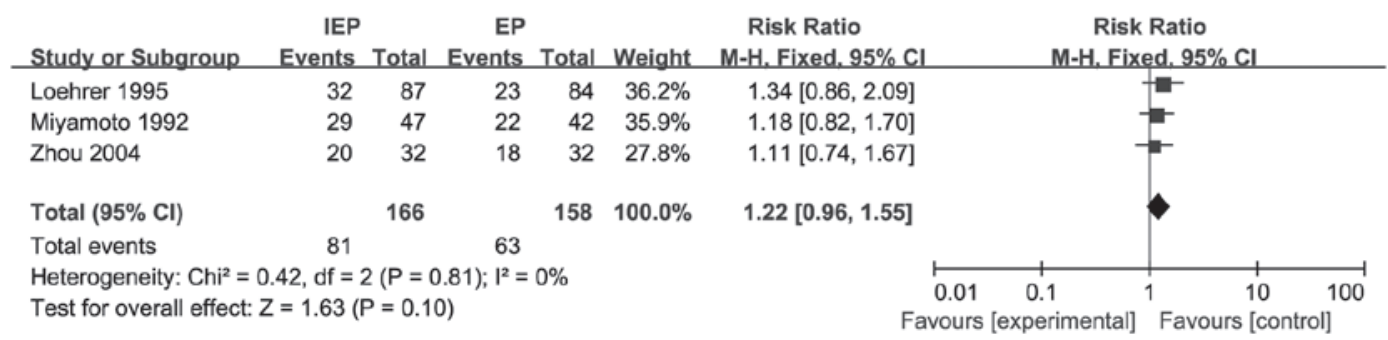

Figure 3. Meta-analysis of 1-year survival rate of patients with small-cell lung cancer who were treated with ifosfamide + etoposide + platinum (IEP) vs. those treated with the etoposide + platinum (EP) regimen. CI, confidence interval.

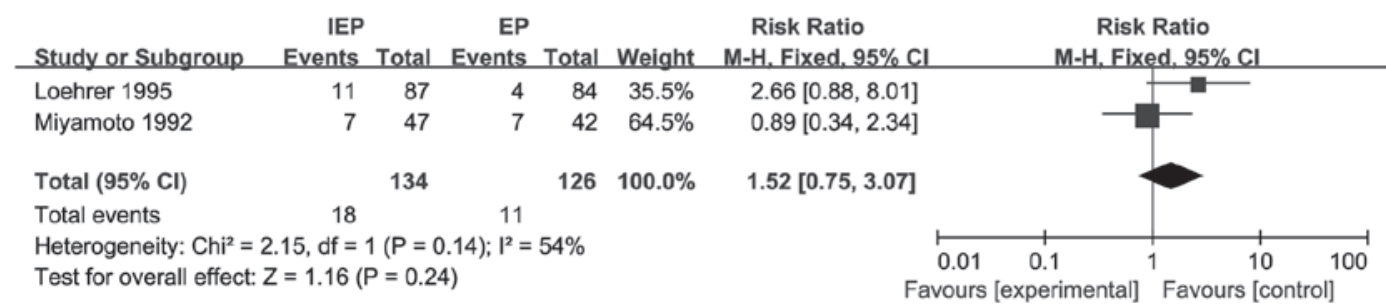

Figure 4. Meta-analysis of 2-year survival rate of patients with small-cell lung cancer who were treated with ifosfamide + etoposide + platinum (IEP) vs. those treated with the etoposide + platinum (EP) regimen. CI, confidence interval.

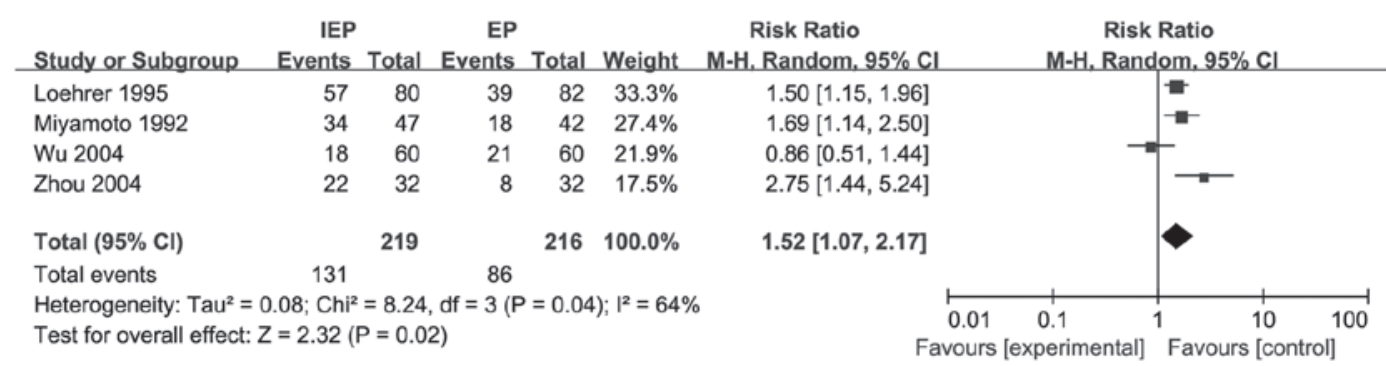

Figure 5. Meta-analysis of hematological toxicity in patients with small-cell lung cancer who were treated with ifosfamide + etoposide + platinum (IEP) vs. those treated with the etoposide + platinum (EP) regimen. CI, confidence interval.

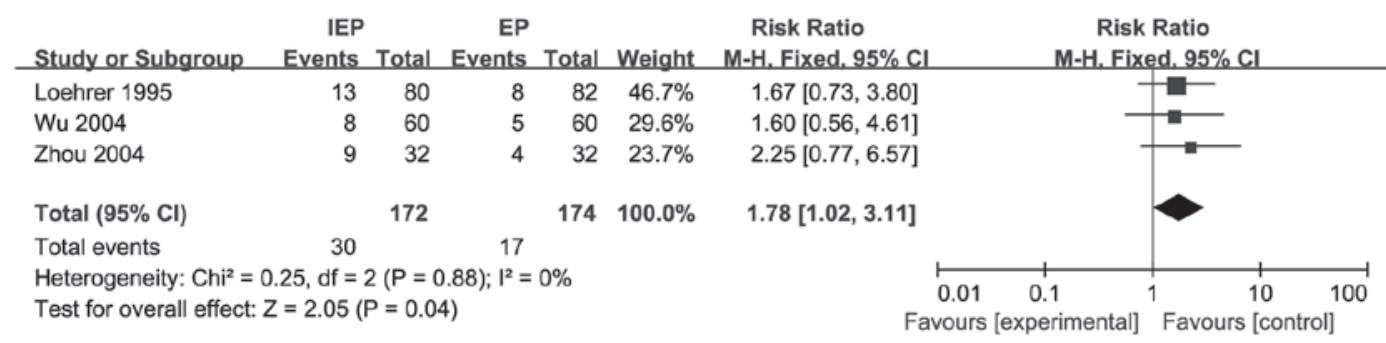

Figure 6. Meta-analysis of gastrointestinal toxicity in patients with small-cell lung cancer who were treated with ifosfamide + etoposide + platinum (IEP) vs. those treated with the etoposide + platinum (EP) regimen. CI, confidence interval. 
EP regimen in terms of overall response; however, the difference was not significant $(\mathrm{RR}=1.07,95 \% \mathrm{CI}$ : 0.97-1.19). There was no heterogeneity $\left(\mathrm{I}^{2}=0 \%, \mathrm{P}=0.50\right)$ and the fixed-effects model was used to pool RR for overall response. The results are shown in Fig. 2.

Survival rate. RRs for 1-year survival rate data were available from 3 trials including 324 patients $(14,15,19)$. The meta-analysis revealed no significant difference $(\mathrm{RR}=1.22$, 95\% CI: 0.96-1.55) between the IEP and EP regimens. There was no heterogeneity $\left(\mathrm{I}^{2}=0 \%, \mathrm{P}=0.81\right)$ and the fixed-effects model was used to obtain the pooled RR for 1-year survival rate. The results are shown in Fig. 3.

RRs for 2-year survival rate data were available from 2 trials including 260 patients $(14,15)$. The pooled RR for the 2-year survival rate revealed that IEP may prolong OS in SCLC patients; however, the difference was not significant ( $\mathrm{RR}=1.52$, 95\% CI: 0.75-3.07). There was significant heterogeneity $\left(\mathrm{I}^{2}=54 \%, \mathrm{P}=0.14\right)$ and the pooled $\mathrm{RR}$ for 2 -year survival rate was calculated using the random-effects model. The results are shown in Fig. 4.

Adverse effects. All 4 trials reported grade 3/4 hematological toxicities and the IEP regimen was more frequently associated with grade 3/4 neutropenia compared to the EP regimen $(\mathrm{R} R=1.52,95 \% \mathrm{CI}: 1.07-2.17)$. There was significant heterogeneity $\left(\mathrm{I}^{2}=64 \%, \mathrm{P}=0.04\right)$ and the pooled $\mathrm{RR}$ for grade $3 / 4$ neutropenia was calculated using the random-effects model. The results are shown in Fig. 5.

Non-hematological toxicities were reported in 3 trials $(15,19,20)$. The pooled data demonstrated that grade $3 / 4$ vomiting was more common with IEP compared to the EP regimen $(\mathrm{RR}=1.78,95 \% \mathrm{CI}: 1.02-3.11)$. There was no heterogeneity $\left(\mathrm{I}^{2}=0 \%, \mathrm{P}=0.88\right)$ and the fixed-effects model was used to obtain the pooled RR for grade 3/4 vomiting. The results are shown in Fig. 6.

\section{Discussion}

Chemotherapy is an essential component of the treatment of all SCLC patients. Although the prognosis of SCLC has improved significantly, long-term survival remains $<10 \%$. According to the American National Comprehensive Cancer Network guidelines, the most common standard first-line therapy for SCLC is the EP regimen (1-5). To obtain an optimal response, more regimens were investigated by adding new drugs to the standard chemotherapy regimen and several clinical trials were conducted to confirm the efficacy and safety of those new regimens in SCLC (9-20).

The IEP regimen is considered as an alternative first-line treatment option for SCLC by an increasing number of physicians; however, it is our opinion that the superiority of IEP to EP requires further clinical confirmation, as different RCTs reported inconsistent results $(14,15,19,20)$. Loehrer et al $(15)$ first reported a group study comparing the IEP to the EP regimen in 163 patients with extensive SCLC and observed significant differences in terms of time-to-progression and OS in favor of the IEP arm. However, Miyamoto et al (14) reported that IEP was not superior to EP chemotherapy in SCLC. Subsequently, Zhou et al (19) and Wu et al (20) further confirmed the results of Loehrer et al in their clinical studies; in addition, Wu et al (20) proved that IEP may also used as salvage chemotherapy for patients with SCLC who failed to respond to EP chemotherapy.

The objective of this meta-analysis was to compare the efficacy and safety of IEP to that of EP in patients with previously untreated SCLC. A total of 4 RCTs involving 447 patients were included. Response rate and grade $3 / 4$ neutropenia were reported in all 4 trials. One-year survival rate was reported by 3 studies and 2-year survival rate by 2 studies. Grade 3 vomiting/nausea were reported by 3 studies. The meta-analysis results demonstrated that IEP failed to achieve a higher response rate and longer survival time, but induced more severe grade $3 / 4$ neutropenia and vomiting compared to the EP regimen.

In conclusion, IEP was not found to be superior to the EP regimen in the first-line treatment of SCLC; in addition, it induced more severe hematological and gastrointestinal toxicities. Therefore, SCLC patients did not obtain any benefit from the addition of ifosfamide to the EP regimen and the role of ifosfamide in multimodality treatment regimens requires further investigation.

\section{References}

1. Jemal A, Siegel R, Ward E, et al: Cancer statistics, 2008. CA Cancer J Clin 58: 71-96, 2008.

2. Masters G: The clinical presentation of small cell lung cancer. In: Lung Cancer: Principles and Practice. Pass HI, Carbone DP, Johnson DH, Minna JD and Turrisi AT III (eds). 3rd edition. Lippincott Williams \& Wilkins, Philadelphia, PA, pp304-314, 2005.

3. Jackman DM and Johnson BE: Small-cell lung cancer. Lancet 366: 1385-1396, 2005.

4. Turrisi AT III, Kim K, Blum R, et al: Twice-daily compared with once-daily thoracic radiotherapy in limited small-cell lung cancer treated concurrently with cisplatin and etoposide. N Engl J Med 340: 265-271, 1999.

5. Sorensen M, Pijls-Johannesma M, Felip E, et al: Small-cell-lung cancer: ESMO Clinical Practice Guidelines for diagnosis, treatment and follow-up. Ann Oncol 21 (Suppl 5): v120-v125, 2010.

6. Sambrook RJ and Girling DJ: A national survey of the chemotherapy regimens used to treat small cell lung cancer (SCLC) in the United Kingdom. Br J Cancer 84: 1447-1452, 2001.

7. Jiang L, Yang KH, Guan QL, et al: Cisplatin plus etoposide versus other platin-based regimens for patients with extensive small-cell lung cancer: a systematic review and meta-analysis of randomised, controlled trials. Intern Med J 42: 1297-1309, 2012.

8. Brade WP, Herdrich K and Varini M: Ifosfamide - pharmacology, safety and therapeutic potential. Cancer Treat Rev 12: 1-47, 1985.

9. Havemann K, Wolf M, Drings P, et al: Experience of a German multicenter study group with ifosfamide in small cell lung cancer. Semin Oncol 16 (Suppl 3): 9-18, 1989.

10. Thatcher N, Cerny T, Stout R, et al: Ifosfamide, etoposide, and thoracic irradiation therapy in 163 patients with unresectable small cell lung cancer. Cancer 60: 2382-2387, 1987.

11. Wolf M, Havemann K, Holle R, et al: Cisplatin/etoposide versus ifosfamide/etoposide combination chemotherapy in small-cell lung cancer: a multicenter German randomized trial. J Clin Oncol 5: 1880-1889, 1987.

12. Evans WK, Stewart DJ, Shepherd FA, et al: VP-16, ifosfamide and cisplatin (VIP) for extensive small cell lung cancer. Eur J Cancer 30A: 299-303, 1994.

13. Loehrer PJ, Rynard S, Ansari R, et al: Etoposide, ifosfamide, and cisplatin in extensive small cell lung cancer. Cancer 69: 669-673, 1992.

14. Miyamoto $\mathrm{H}$, Nakabayashi $\mathrm{T}$, Isobe $\mathrm{H}$, et al: A phase III comparison of etoposide/cisplatin with or without added ifosfamide in small-cell lung cancer. Oncology 49: 431-435, 1992.

15. Loehrer PJ Sr, Ansari R, Gonin R, et al: Cisplatin plus etoposide with and without ifosfamide in extensive small-cell lung cancer: a Hoosier Oncology Group study. J Clin Oncol 13: 2594-2599, 1995. 
16. Wolff AC, Ettinger DS, Neuberg D, et al: Phase II study of ifosfamide, carboplatin, and oral etoposide chemotherapy for extensive-disease small-cell lung cancer: an Eastern Cooperative Oncology Group pilot study. J Clin Oncol 13: 1615-1622, 1995.

17. Glisson B, Lee JS, Palmer J, et al: Cisplatin, ifosfamide, and prolonged oral etoposide in the treatment of patients with extensive small cell lung carcinoma. Cancer 82: 301-308, 1998.

18. Hand S, Baker J, Smith AP, et al: Outpatient intensive chemotherapy for small cell lung cancer: five years experience of modified 'ICE' ifosfamide carboplatin and etoposide. Clin Oncol (R Coll Radiol) 14: 367-371, 2002.
19. Zhou H, Wang A, Huang Z, et al: Randomized clinical trial of IEP and EP regimens in the treatment of patients with small cell lung cancer. Chin J Lung Cancer 7: 240-242, 2004 (In Chinese).

20. Wu C, Qi H, Dai Y, et al: Therapeutic efficacy of chemotherapy with VIP for small cell lung cancer. Chin J Lung Cancer 7: 151-153, 2004 (In Chinese). 